Pacific Journal of Mathematics

HARMONIC FUNCTIONS WITH ARBITRARY LOCAL 


\title{
HARMONIC FUNCTIONS WITH ARBITRARY LOCAL SINGULARITIES
}

\author{
William C. Fox
}

1. Introduction. This paper is concerned with a new and more informative solution of an old existance problem, that of determining what conditions must be imposed upon the nature of a local harmonic singularity in order to imply the global existence of a harmonic function which "has" the given singularity. In 1870, [17, vol. II, p. 133143 and p. 144-177], H. A. Schwarz solved the problem for closed surfaces giving, as sufficient, the condition that the harmonic singularity function must have vanishing flux across the curve (bounday of a disk) on which it is given, and he solved the problem for open surfaces which are interiors of compact manifolds-with-boundaries, with no restriction on the singularity function. In 1909 [4, vol. 3, p. 73-80] Hilbert announced that the problem for open surfaces, with singularities having flux, can be solved by a special extremal method. Hilbert worked out an illustrative example and left the general account to be presented in the thesis of his student Richard Courant. A few months later Koebe [6], in the last of his series of four papers on the uniformization of analytic curves, gave the first full account of the existence of harmonic functions with a prescribed local singularity on open surfaces. Koebe based his proofs on exhaustion and the results of Schwarz; he did not use Hilbert's special extremal method. Moreover, his convergence arguments still used the assumption that the singularity's flux is zero. In 1910 and 1912 $[2,3]$ Courant published accounts of special cases taken from his thesis; not again did Hilbert's special extremal method appear in print. In 1913, Weyl [20] re-proved Koebe's theorem using an extremal method, namely that of minimizing the Dirichlet integral of what he called the "concurrence functions." (In all these works the singularity function was specified in concrete terms, e.g., as the real part of $1 / z^{n}$ near the origin. However, the proofs remain valid for any singularity with vanishing flux. Accordingly, I have described them in those terms.)

Not until 1953 were any further advances made with respect to this existence problem. At that time, Sario [13] published a modern account (based on preliminary notes dated 1949 and 1950) of the alternating series method of Schwarz which went far beyond the work of Schwarz both in method and in generality. When Sario's results are

Received March 15, 1960. Research which led to this paper was supported in part by the National Science Foundation. A preliminary report was made at the 1957 summer meeting of the American Mathematical Society. 
restricted to the case of a local singularity, they duplicate those of Schwarz for closed surfaces and in the case of open surfaces they relieve Koebe's theorem of the vanishing flux restriction on the singularity. Sario states further that when the flux does vanish one may conclude that the function asserted to exist is bounded and has finite Dirichlet integral on any domain on whose closure it is harmonic.

In this paper, Sario's results are sharpened in various ways. Among others: a necessary and sufficient condition for the flux to vanish is that a potential function exists which "has" the prescribed singularity and whose normal derivatives vanish (in a certain strong sense) on the ideal boundary. On open surfaces, there always exists a potential function which "has" the prescribed singularity and which vanishes on the ideal boundary. These conditions (on the functions' behavior at the ideal boundary) determine the two potential functions uniquely (up to additive constants) as solutions to certain extremal problems. Concerning either of these two potential functions, one may always state that it is bounded and of finite Dirichlet integral on any domain on whose closure it is harmonic, even when the singularity's flux is not zero. Moreover, the extremal properties shed some light on the role of Sario's assumption that the given singularity function, harmonic on certain Jordan curves, vanishes on these curves.

An alternative existence proof is given here also. Its preliminary part (Theorem 1) on uniform boundedness is of some intrinsic interest. Although it parallels Sario's Lemma 3 [13, p. 636], it was suggested by similar arguments used by Koebe in 1909 [6] and in his 1910 recapitulations [7, 8]. Mainly, however, the alternative existence proof is given here because it also yields the other results described above.

2. Uniform boundedness. The existence theorem of the next section makes use of the Ascoli theorem. For this purpose some information is needed on the existence of a uniform bound for certain families of potential functions. In what follows, the term "local coordinate" refers to any homeomorphism (from a domain in the sphere onto a domain in the Riemann surface in question) which is also analytic.

Theorem 1. In the Riemann surface $X$, let $B_{I} \subset B_{0}$ be the images under a local coordinate of concentric open disks, and let $S$ be harmonic on the closed "annulus" $\left(B_{0}-B_{I}\right)^{-}$. If $\mathscr{U}$ is a set of functions, $u$, with each of which is associated a domain $D(u)$ in $X$ containing $\left(B_{0}\right)^{-}$such that

(i) each function $u$ is harmonic on $D(u)-B_{I}$ and is bounded there by its extreme values on the boundary of $B_{I}$,

(ii) each function $u-S$ determines a harmonic function $U$ on $\left(B_{0}\right)^{-}$which agrees with $u-S$ on $B_{0}-B_{I}$, and 
(iii) for some point $Q$ of $B_{I}$ the set of values $\{U(Q): u \in \mathscr{U}\}$ is bounded,

then there exists a constant $K$ such that $|u| \leqq K$ on $D(u)-B_{I}$ for every $u$ in $\mathscr{U}$.

Proof. Let $M(u)$ denote the maximum value of $|u|$ on the boundary of the inner disk $B_{I}$, by hypotheses, the relation $|u| \leqq M(u)$ holds on $D(u)-B_{I}$ and there then must exist a point $p(u)$ in the boundary of $B_{I}$ at which $\mid u(p)=M(u)$. If the set $\{M(u): u \in \mathscr{U}$ is bounded above then the theorem is proved. Otherwise, there exists a sequence $\left\{u_{n}\right\}$ in $\mathscr{U}$ such that (2.1) $\lim _{n} M\left(u_{n}\right)=+\infty$ and (2.2) $\lim _{n} p\left(u_{n}\right)-p$. On the outer disk $B_{0}$, the function $U_{n}$ (which agrees with $u_{n}-S$ on $B_{0}-B_{I}$ ) is harmonic and so is bounded there by its extreme values on the boundary of $B_{0}$, so that

$$
\left|U_{n}\right| \leqq \operatorname{lub}\left\{\left|u_{n}(x)\right|: x \in \partial B_{0}\right\}+\operatorname{lub}\left\{|S(x)|: x \in \partial B_{0}\right\} .
$$

If the right hand term in this inequality is abbreviated by the symbol $M$, then relation (2.3), by hypotheses, may be written in the form

$$
\left|U_{n}\right| \leqq M\left(u_{n}\right)+M \text { on } B_{0} .
$$

Thus

$$
\frac{\left|U_{n}-U_{n}(Q)\right|}{M\left(u_{n}\right)} \leqq 2\left(1+\frac{M}{M\left(u_{n}\right)}\right) \text { on } B_{0}
$$

By (2.1), this implies that the sequence $\left(U_{n}-U_{n}(Q)\right) / M\left(u_{n}\right)$ is uniformly bounded on $B_{0}$. Moreover, the sequence $u_{n} / M\left(u_{u}\right)$ is uniformly bounded on the ring $\left(B_{0}-B_{I}\right)^{-}$. The Ascoli theorem guarantees the existence of a subsequence of indeces for which the following limits exist uniformly on the domains indicated:

$$
\lim _{n} \frac{U_{n}-U_{n}(Q)}{M\left(u_{n}\right)}=H_{0} \text {, harmonic on } B_{0},
$$

(2.7) $\lim _{n} \frac{u_{n}}{M\left(u_{n}\right)}=H$, harmonic on $B_{0}-\left(B_{I}^{-}\right)$, continuous on $\left(B_{0}-B_{I}\right)^{-}$.

By hypotheses the sequence of numbers $\left\{U_{n}(Q)\right\}$ is bounded. Thus by (2.1), one may conclude that

$$
\lim _{n} \frac{S}{M\left(u_{n}\right)}=0 \text { and } \lim _{n} \frac{U_{n}(Q)}{M\left(u_{n}\right)}=0
$$

whence

(2.9) $H_{0}=\lim _{n} \frac{u_{n}}{M\left(u_{n}\right)}-\lim _{n} \frac{S}{M\left(u_{n}\right)}-\lim _{n} \frac{U_{n}(Q)}{M\left(u_{n}\right)}=H$ on $B_{0}-B_{I}$. 
It is now necessary to show that $H_{0}(p)=1$ when $p$ is given by (2.2). Let $p_{n}$ denote $p\left(u_{n}\right)$ and let $a_{n j}=\left|u_{n}\left(p_{j}\right) / M\left(u_{n}\right)\right|$. In view of the facts.

$$
\left\{\begin{array}{l}
\lim _{n} a_{n j}=\left|H\left(p_{j}\right)\right| \text { uniformly in } j, \text { and } \\
\lim _{j} a_{n j}=\left|u_{n}(p)\right| M\left(u_{n}\right) \mid \text { for each } n
\end{array}\right.
$$

one may apply the Moore-Smith theorem on iterated limits. It follows

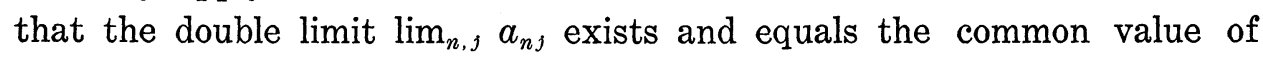
the two iterated limits:

$$
\lim _{n}\left(\lim _{j} a_{n j}\right)=\lim _{j}\left(\lim _{n} a_{n j}\right)=H_{0}(p) .
$$

(The last equality follows from (2.8) and (2.9), since the points $p_{n}$ are all in $B_{0}-B_{I}$, for that set contains the boundary of $B_{I}$.) Since $a_{n n}=1$ for all $n$ (by definition of $p_{n}$ ), it is the case that $\left|H_{0}(p)\right|=1$. Since $H_{0}(Q)=0$, this means that $H_{0}$ is not constant on $B_{0}$.

On the other hand, $H_{0}$ must be constant, in virtue of the following considerations. Restricted to $B_{I}, H_{0}$ certainly is bounded by its extreme values on the boundary of $B_{I}$. Restricted to the ring $B_{0}-B_{I}$, it is still true that $H_{0}$ is bounded by its extreme values on the boundary of $B_{I}$ because $H_{0}$ inherits this property from the sequence $u_{n} / M\left(u_{n}\right)$ which by (2.8) and (2.9) converges to $H_{0}$ on $B_{0}-B_{I}$. This means that $H_{0}$ must have a local maximum at some point of the boundary of $B_{I}$, necessarily an interior point of $B_{0}$, whence $H_{0}$ is constant. This contradiction completes the proof.

3. The existence theorem. The method of exhaustion requires that the existence problem be solved on subdomains with compact closure and smooth boundaries. For the present purposes this was started by H. A. Schwarz in 1870 and was completed by Koebe in 1910, [8]. A portion of the proof is sketched here to indicate how the hypotheses enter the arguments.

If $r$ and $s$ are $C^{\prime}$ on the interior of $Y$ except for a closed set of measure zero then the Dirichlet integral of $r$ relative to $s$ over $Y$ will be denoted by $\mathfrak{D}(r, s ; Y)$. When $r=s$, the Dirichlet integral will be written simply $\mathfrak{D}(r ; Y)$. When $Y$ is a plane domain, one has

$$
\mathfrak{D}(r, s ; Y)=\int_{Y}\left(\frac{\partial r}{\partial x} \frac{\partial s}{\partial x}+\frac{\partial r}{\partial y} \frac{\partial s}{\partial y}\right) d \mu
$$

where $\mu$ is Lebesgue measure in $Y$.

Lemma. Let $X^{-}$be a compact two-manifold-with-boundary and let its interior, $X \neq X^{-}$, be a Riemann surface. If $B_{I} \subset B_{0}$ are images 
under a local coordinate in $X$ of concentric open disks and if $S$ is harmonic on the closed "annulus" $\left(B_{0}-B_{I}\right)^{-} \subset X$, then there exists a function $u$, and when $S$ has a single-valued conjugate, there exists another function $v$, such that

(i) they are harmonic on $X-B_{I}$,

(ii) their differences with $S$ have harmonic extensions from $B_{0}-B_{I}$ to $B_{0}$,

(iii) they are bounded on $X-B_{0}$ by their extreme values on the boundary of $B_{0}$,

(iv) $\mathfrak{D}\left(v, T ; X-B_{0}\right)=+\int_{\partial B_{0}} T d v^{*}$ for every function $T, C^{\prime}$ on $X-B_{0} ;$ and

(v) $\mathfrak{D}\left(u, H ; X-B_{0}\right)=+\int_{\partial B_{0}} u d H^{*}$ for every function $H$ harmonic on $X-B_{0}$.

Proof. By definition of a two-manifold-with-boundary, every boundary point of $X^{-}$is contained in a Jordan curve one of whose complementary domains in $X$ is simply connected. By the Osgood-Caratheodory extension of the Riemann mapping function, there exists a homeomorphism of this domain's closure onto the closed unit disk, a homeomorphism which is analytic on the domain itself and which sends an arc of the boundary of $X$ onto an arc of the unit circle. Thus, it is no restriction to suppose that each component of the boundary of $X$ is an analytic Jordan curve, which in turn makes it possible to form the "double" of $X^{-}$. (Of course, when $X^{-}$is contained in some larger Riemann surface $Y$ this argument does not imply that the boundary of $X$ is analytic in $Y$, but only analytic relative to the coordinate system of $X$ itself.)

Let $X^{*}$ denote the double of $X^{-}$; there is then an analytic homeomorphism, a reflection, $R$ of $X^{*}$ onto itself which leaves the boundary of $X$ pointwise fixed and which sends $X$ onto $X^{*}-X^{-}$. Let $B_{0}^{*}=R\left(B_{0}\right)$, $B_{I}^{*}=R\left(B_{I}\right)$ and $S^{*}=S\left(R^{-1}\right)$. Both $S$ and $S^{*}$, by hypothesis, have single-valued harmonic conjugates on the annuluses $B_{0}-B_{I}$ and $B_{0}^{*}-B_{I}$ respectively. Since $X^{*}$ is a closed surface, this condition is needed to warrant the conclusion that a function $v^{*}$, harmonic on $X^{*}-\left(B_{I} \cup B_{I}^{*}\right)$, exists such that $v^{*}-S$ and $v^{*}-S^{*}$ have harmonic extensions to $B_{0}$ and $B_{0}^{*}$ respectively. Moreover these conditions make $v^{*}$ unique up to an additive constant since the only functions harmonic on a closed surface are the constants. Therefore $v^{*}(R)-v^{*}$ is not only constant but is zero because $R$ leaves the (non-empty) boundary of $X$ pointwise fixed. This implies that $v^{*}$ is bounded on $X^{*}-\left(B_{0} \cup B_{0}^{*}\right)$ by its extreme values on the boundary of $B_{0}$ alone, for they are related by the reflection $R$ to those on the boundary of $B_{0}^{*}$. Of course the normal derivatives of 
$v^{*}$ vanish on the boundary of $X$, also because of the relation $v^{*}(R)-v^{*}=0$ and the fact that, on neighborhoods of points of the boundary of $X, R$ is literally a reflection. Thus, if $v$ denotes the restriction of $v^{*}$ to $X^{-}-B_{I}$ then (i), (ii) and (iii) have been proved, and (iv) is a consequence of the Green's formula. This use of the "double" of $X$ is due to Koebe [8].

To construct $u$, one applies the existence theorem of Schwarz (for open surfaces) with boundary values being the constant zero. Properties (i) and (ii) are then immediate; (v) is a consequence of the Green's formula and the boundary values of $u$, and the latter implies (iii) also.

It is now possible to prove the main theorem.

TheOREM 2, PART I: Existence. Let $X$ be a Riemann surface, let $B$ and $\beta$ be images under a local coordinate in $X$ of an open disk and its boundary respectively, and let $S$ be harmonic on $\beta$. A necessary and sufficient condition that $\int_{\beta} d S^{*}=0$ is that there exists a real function $v$

(i) which is a potential function, on $X$, whose singularity is $S$, (i.e., which is harmonic on $X-B$ and whose difference with $S$ has a harmonic extension to $B^{-}$).

and

(ii) which is bounded on $X-B$ by its extreme values on $\beta$,

(iii) for which $d v^{*}=0$ on $\partial X$, i.e.,

$$
\int_{\partial X} w d v^{*}=\mathfrak{D}(w, v ; X-B)-\int_{\beta} w d v^{*}=0
$$

for every function $w, C^{\prime}$ on $X-B^{-}$and continuous on the closure.

If $S$ is an arbitrary harmonic function on $\beta$ and if $X$ is open, there always exists a potential function, $u$, on $X$, whose singularity is $S$, which has property (ii) and

(iv) for which $u=0$ on $\partial X$, i.e.,

$$
\int_{\partial X} u d w^{*}=\mathfrak{D}(u, w ; X-B)-\int_{\beta} u d w^{*}=0
$$

for every function $w$ harmonic on $X-B$.

TheOREM 2, PART II: Uniqueness: When $v$ exists there then also exists a function $r$, harmonic on $B^{-}$, for which $d(S-r)^{*}=0$ on $\beta$; whether or not $v$ exists, there always exists a function $s$, harmonic on $B^{-}$, for which $S-s=0$ on $\beta$. The functions $v$ and $u$ are determined uniquely up to an additive constant, among all potential functions on 
$X$ whose singularities are $S$, by their respective properties (iii) and (iv) as the functions for which:

(v) The quantity $\int_{\beta}(S-r) d w^{*}+\int_{\partial X} d w^{*}$ is minimized by $w=v$ among all functions $w$, harmonic on $X-B$.

(vi) The quantity $\int_{\beta} w d(S-s)^{*}+\int_{\partial X} w d w^{*}$ is minimized by $w=u$ among all functions $w$ harmonic on $X-B$.

In each case the minimum values are $\int_{\beta}(S-r) d v^{*}$ and $\int_{\beta} u d(S-s)^{*}$ respectively.

Proof. Let $B=B_{0}$ and let $B_{I}$ be concentric with $B_{0}$ such that $S$ is harmonic on $\left(B_{0}-B_{I}\right)^{-}$. Let $\left\{D_{n}\right\}$ be an exhaustion of $X$ such that $\left(B_{0}\right)^{-} \subset D_{I}$, i.e., $\left\{D_{n}\right\}$ is a sequence of domains, the union of which is $X$, each with compact closure and with boundary consisting of sectionally analytic Jordan curves. By the lemma, there exists a potential function $u_{n}$, on $D_{n}$ whose singularity is $S$, which vanishes continuously on the boundary of $D_{n}$, and is bounded, by its extreme values on $\beta$, on $D_{n}-B_{0}$. Let $Q$ is a point in $B_{I}$ and let $a_{n}$ be the value taken at $Q$ by the harmonic extension to $B_{0}$ of $u_{n}-S$. If $\left\{a_{n}\right\}$ is an unbounded sequence let $\left\{u_{n}\right\}$ be replaced by $\left\{u_{n}-a_{n}\right\}$. By Theorem 1 the sequence $\left\{u_{n}\right\}$ is uniformly bounded on each set $D_{n}-B_{I}$ and so contains a subsequence which converges uniformly on each set $D_{n}-B_{I}$ to a function $u$ which then inherits properties (i) and (ii).

NoтE. When each $u_{n}$ is the Green's function of $D_{n}$ with pole at $Q$ then $a_{n}$ is called the principle part of $u_{n}$ and the sequence $\left\{a_{n}\right\}$ is necessarily monotone increasing. By use of Harnack's theorem one sees that $\left\{a_{n}\right\}$ is bounded above if and only if $u$ is the Green's function for $X$. This characterization of the Green's function's existence (the convergence of a sequence of principle parts) was first discovered by Koebe in his proof of the so-called uniformization theorem [9], when $X$ is simply connected.

By. (v) in the lemma, one may establish the following relations, once $u_{n}$ has been extended continuously to $X-D_{n}$ to be constant there:

$$
\left\{\begin{aligned}
\mathfrak{D}\left(u, H ; X-B_{0}\right) & =\lim _{n} \mathfrak{D}\left(u_{n}, H ; X-B_{0}\right) \\
& =\lim _{n} \mathfrak{D}\left(u_{n}, H ; D-B_{0}\right) \\
& =\lim _{n} \int_{\beta} u_{n} d H^{*}=\int_{\beta} u d H^{*} .
\end{aligned}\right.
$$

This establishes (iv) here. Note that the additive constants $a_{n}$, should they be present, do not have any effect, for they disappear in the integrand of the Dirichlet integral.

If $S$ does have a single-valued harmonic conjugate, i.e., if

$$
\int d S^{*}=0
$$


then the corresponding functions $v_{n}$ certainly exist, by the lemma, and so therefore does $v$ by Theorem 1 . Moreover

$$
\left\{\begin{aligned}
\mathfrak{D}\left(v, T ; X-B_{0}\right) & =\lim _{n} \mathfrak{D}\left(v_{n}, T ; X-B_{0}\right) \\
& =\lim _{n} \mathfrak{P}\left(v_{n}, T ; D-B_{0}\right) \\
& =\lim _{n} \int_{\beta} T d v_{n}^{*}=\int_{\beta} T d v^{*},
\end{aligned}\right.
$$

as required by (iii). In (3.1) and (3.2), one needs to know that the partial derivatives of $u_{n}$ and $v_{n}$ will converge to those of $u$ and $v$. Conversely, given (iii), one may choose $w=1$ and obtain

$$
\int_{\beta} d v^{*}=0 \text {. }
$$

Since $v-S$ has a harmonic extension to $\left(B_{0}\right)^{-}$and therefore has a single-valued harmonic conjugate there, it follows that

$$
\int_{\beta} d(v-S)^{*}=0,
$$

whence

$$
\int_{\beta} d S^{*}=0
$$

It is worth observing that the choices $T=v$ and $H=u$ lead to the conclusions

$$
\left\{\begin{array}{l}
\mathfrak{D}\left(u ; X-B_{I}\right)=\int_{\beta} u d u^{*}, \text { and } \\
\mathfrak{D}\left(v ; X-B_{I}\right)=\int_{\beta} v d v^{*} .
\end{array}\right.
$$

Thus both $u$ and $v$ have finite Dirichlet integrals over $X-B_{0}$. Moreover, according to (iii) $v$ has the property that $\mathfrak{D}\left(v, T ; X-B_{0}\right)$ is finite even for functions $T$ such that $\mathfrak{D}\left(T ; X-B_{0}\right)$ is not finite. A similar remark holds for $u$.

Using the notation

$$
\mathfrak{D}\left(w ; X-B_{0}\right)=\int_{\beta} w d w^{*}+\int_{\partial X} w d w^{*}
$$

for harmonic $w$, with the above observations, one may verify that

$$
\int_{\partial X} u d u^{*}=0 \text { and } \int_{\partial X} v d v^{*}=0,
$$

facts which will be used below.

Let $t$ be a function harmonic on $B^{-}$. To prove part II one must establish that the quantities to be minimized in (v) and (vi) exhibit 
quadratic-form properties. For this purpose it is convenient to introduce the notion of an $(S-t)$-concurrence function as a function $W, C^{\prime}$ on $X-\beta$ for which

$$
W+(S-t) \chi_{x-B^{-}}
$$

is continuous on a neighborhood of $\beta$. (Of course, $\chi_{z}$ denotes the characteristic function of $Z$.) If $w$ belongs to the class $\mathscr{C}$ of all functions $C^{\prime}$ on $X-B^{-}$and continuous on the closure, then $w$ determines an $(S-t)$-concurrence function, denoted by $W_{S-t}$, by the rule that $W_{S-t}=w$ on $X-B^{-}$and $W_{S-t}$ is, on $B^{-}$, the harmonic function determined by the boundary values $w-(S-t)$. Thus, every $W_{s-t}$ is harmonic on $B^{-}$. If $y$ is a potential function on $X$ whose singularity is $S$ and $w$ is an arbitrary member of $\mathscr{C}$ then

$$
\left\{\begin{array}{l}
\mathfrak{D}\left(Y_{S-t} ; Y_{S-t}-W_{S-t} ; B\right)=-\int_{\beta}(y-w) d(y-(S-t))^{*} \\
\mathfrak{D}\left(Y_{S-t} ; Y_{S-t}-W_{S-t} ; X-B\right)=\int_{\beta}(y-w) d y^{*}+\int_{\partial X}(y-w) d y^{*}, \text { and } \\
\mathfrak{D}\left(Y_{S-t}, Y_{X-t}-W_{S-t} ; X\right)=\int_{\beta}(y-w) d(S-t)^{*}+\int_{\partial X}(y-w) d y^{*}
\end{array}\right.
$$

If $w$ is a member of the class $\mathscr{H}$ of all functions harmonic on $X-B$, then

$$
\left\{\begin{array}{l}
\mathfrak{D}\left(Y_{S-t}, Y_{S-t}-W_{X-t} ; B\right)=-\int_{\beta}(y-(S-t)) d(y-w)^{*}, \\
\mathfrak{D}\left(Y_{S-t}, Y_{S-t}-W_{S-t} ; X-B\right)=\int_{\beta} y d(y-w)^{*}+\int_{\partial X} y d(y-w)^{*}, \text { and } \\
\mathfrak{D}\left(Y_{S-t}, Y_{X-t}-W_{S-t} ; X\right)=\int_{\beta}(S-t) d(y-w)^{*}+\int_{\partial X} y d(y-w)^{*} .
\end{array}\right.
$$

These relations are consequences of the Green's formula and the facts that every $W_{s-t}$ takes, on $\beta$, the values $w-(S-t)$ and $w$ according as one approaches $\beta$ from $B$ or from $X-B^{-}$and the difference of two $(S-t)$-concurrence functions is $C^{\prime}$ on all of $X$. Therefore

The Dirichlet-variation $\mathfrak{D}\left(Y_{S-t}, Y_{S-t}-W_{S-t} ; X\right)$ vanishes in each of the following cases:
(a) when $y=v$ and $w-y=0$ on $\beta$ and on $\partial X$,
(b) when $y=v$ and $t=r$
(c) when $y=u$ and $d(w-y)^{*}=0$ on $\beta$ and on $\partial X$, and
(d) when $y=u$ and $t=s$.

Cases (a) and (c) are immediate, whereas (b) and (d) are consequence of (iii) and (iv), and the properties of $r$ and of $s$.

The quadratic form character of the Dirichlet integral makes it easy to verify that 


$$
\left\{\begin{array}{l}
\mathfrak{D}(a, a-b ; Z)=0 \text { if and only if } \\
\mathfrak{D}(b ; Z)-\mathfrak{D}(a ; Z)=\mathfrak{D}(b-a ; Z) .
\end{array}\right.
$$

Since $\mathfrak{D}(b-a ; Z)$ is non-negative the vanishing of the Dirichlet-variations in (3.8) are equivalent, respectively, with the following:

$$
\begin{aligned}
& \left(()^{\prime} \quad \mathfrak{D}\left(V_{s-t} ; X\right) \leqq \mathfrak{D}\left(W_{s-t} ; X\right) \text { for all } w \text { in } \mathscr{C} \text { for which } w-v=0\right. \\
& \text { on } \beta \text { and on } \partial X \text {, } \\
& \text { (b)' } \mathfrak{D}\left(V_{S-r} ; X\right) \leqq \mathfrak{D}\left(W_{S-r} ; X\right) \text { for all } w \text { in } \mathscr{C} \\
& \text { (c)' } \mathfrak{D}\left(U_{s-t} ; X\right) \leqq \mathfrak{D}\left(W_{S-t} ; X\right) \text { for all } w \text { in } \mathscr{H} \text { for which } \\
& d(w-u)^{*}=0 \text { on } \beta \text { and on } \partial X \text {, and } \\
& (\mathrm{d})^{\prime} \quad \mathfrak{D}\left(U_{S-t} ; X\right) \leqq \mathfrak{D}\left(W_{S-s} ; X\right) \text { for all } w \text { in } \mathscr{H} \text {. }
\end{aligned}
$$

In each of these cases, $\mathfrak{D}(b-a ; Z)=0$ implies that $b-a$ is constant, which establishes the uniqueness claims. The extremal properties stated in (v) and (vi) are derived from the inequalities (b)', and (d)' by expanding both sides. In general, when $w$ is in $\mathscr{H}$,

$$
\left\{\begin{aligned}
\mathfrak{D}\left(W_{S-t} ; X\right)= & \mathscr{D}\left(W_{S-t} ; B\right)+\left(W_{S-t}, X-B\right) \\
= & -\int_{\beta}(w-(S-t)) d(w-(S-t))^{*}+\int_{\beta} w d w^{*}+\int_{\partial X} w d w^{*} \\
= & +\int_{\beta} w d(S-t)+\int_{\beta}(S-t) d w^{*}-\int_{\beta}(S-t) d(S-t)^{*} \\
& +\int_{\partial X} w d w^{*} .
\end{aligned}\right.
$$

In (a)', the first and third terms, above, are common to both sides; in (b)' those terms both vanish since $d(S-r)^{*}=0$ on $\beta$. In (c)' the second and third terms, above, are common to both sides, and in (d)' these become zero because $S-s=0$ on $\beta$.

The extremal properties of (a)' and (c)' are of no interest because any two of the harmonic functions involved must differ by a constant. Whereas $v$ and $u$ were shown to solve (b)' and (d)' by use of (iii) and (iv), they were shown to solve (a)' and (c)' automatically. Hence any other of the competing functions also solves (a)' or (c)', whence the competing functions all differ from $u$ (or $v$ ) by constants.

It remains only to verify the existence of $r$ and $s$. By the Poisson formula there is a function $s$, harmonic on $B_{0}$, given by the boundary values $S$. Since $S-s=0$ on the boundary, $\beta$, of $B_{0}$, it may be continued across the the boundary by reflection. Therefore, $s$ is harmonic on $\left(B_{0}\right)^{-}$because $S$ is harmonic on $\left(B_{0}-B_{I}\right)^{-}$. When $S$ is the real part of a complex function $S+i T$ analytic on $\left(B_{0}\right)^{-}$then in a like manner one may construct a function $t$, harmonic on $\left(B_{0}\right)^{-}$, which agrees with $T$ on the boundary. Since $B_{0}$ is a disk, $t$ is the imaginary part of a complex function $r+i t$ analytic on $\left(B_{0}\right)^{-}$. Since $T-t=0$ on the bound- 
ary of $B_{0}$ and is conjugate to $S-r$, it follows that $d(S-r)^{*}=0$ on the boundary of $B_{0}$ also, as required to complete the proof.

The evaluation of $\mathfrak{D}\left(W_{S-t} ; X\right)$ in (3.11) relies upon the assumption that $w$ is harmonic on $X-B$ so that the Green's formula may be used there. This restriction makes it possible to phrase (v) in a compact form, though it does cause that statement to be incomplete. However, the supply of functions; harmonic on $X-B$ is sufficiently ample to make it possible for the existence of $v$ to be proved by a direct approach to the extremal problem (v) rather than by exhaustion. (Indeed, except for the restriction to harmonic functions, this is exactly how Weyl's existence proof was accomplished, for he minimized the Dirichlet integral of all $(S-r)$-concurrence functions.) This fact makes one suspect that it may be possible to prove the existence of $u$ by a direct approach to the extremal problem (vi). I will discuss this possibility in another paper.

From (3.9) and the expansion in (3.6) it is clear that

$$
\mathfrak{D}(y, X-B) \geqq \mathfrak{D}(w ; X-B)=\int_{\beta} w d w^{*}+\int_{\partial X} w d w^{*}
$$

for every $w$ harmonic on $X-B$ for which $w=y$ on $\beta$ and on $\partial x$. Sario $[14$, p. 354] has discovered that, when $y=v$, the requirement " $w=y$ on $\partial X$ " may be replaced by the requirement " $\int_{\beta} d w^{*}=0$ " which is of course equivalent with " $\int_{\partial X} d w^{*}=0$ ", and (3.12) continues to hold. He uses this fact to show that the existence of $v$ implies $\int_{\beta} d S^{*}=0$, whereas in the present discussion property (iii) is used for this purpose as well as to characterize $v$ uniquely up to an additive constant. The function $v$ is Sario's "principal function $P_{-1}^{0}$." By (iii), when $y=v$, the relation " $w=y$ on $\partial X$ " holds for every $C$ ' function $w$, so that Sario's condition " $\int_{\beta} d w^{*}=0$ " is not necessary, for the extremal property (3.12) itself. The fact that (3.12) holds for all $w$ which agree with $v$ on $\beta$ regardless of their behavior "at infinity" was known to Hilbert in 1909 [4, vol. 3, p. 78].

The existence of $v$ was announced first by Hilbert [4, vol. 3, pp. 73-80] and fully proved first by Koebe [6]. Its extremal property (v) was discovered and proved by Weyl [20]. The existence of $u$ with property (i) was proved first by Sario [13]. Properties (ii), (iv) and (v) for $u$ are new, as well as their unique determination of $u$.

The results given here may be generalized along the lines of Sario's linear operators $[14,15]$ by consideration of more general domains for the singularity function $S$ and of extremal properties involving other combinations of the quantities appearing in (v) and (vi). Alternatively, once the existence of potential functions with an arbitrary local singularity has been settled (as in the present theorem 2) one may build a 
sequence of potential functions each with local singularity in one of a sequence of "localities" and then combine them with coefficients which force convergence of the resulting series. Such a technique was first proposed by Koebe [10] in his proof that every open surface is conformally equivalent with a continuation manifold (needed to fill a gap in his first proof of the so-called uniformization theorem); a more detailed version was given by Stoilow [18, p. 59-60].

\section{BIBLIOGRAPHY}

1. Constantin Caratheodory, Gesammelte Mathematische Schriften, C. H. Beck, 1955.

2. Richard Courant, Zur Begrundung des Dirichletschen Prinzipes, Göttingen Nachrichten, (1910), 154-160.

3. - Uber die Methode des Dirichletschen Prinzipes, Mathematische Annalen, 72 (1912), 517-550.

4. David Hilbert, Gesammelte Abhandlungen, Springer, 1935.

5. O. D. Kellogg, Foundations of Potential Theory, Frederick Ungar, 1929.

6. R. Koebe, Uber die Uniformisierung beliebieger analytischer Kurven, Göttingen Nachrichten, (1909), 324-361.

7. — Uber die Uniformisierung beliebieger analytischer Kurven, Journal fur die: reine und angewandte Mathematik, 138 (1910), 192-253.

8. — Uber die Hilbertsche Uniformisierungsmethode. Göttingen Nachrichten, 1910,. 59-74.

9. U_ Uber die Uniformisierung beliebieger analytischer Kurven, Göttingen Nach-richten, (1907), 191-210.

10. - Fonction potentialle et fonction analytique ayant un domaine d'existence: donné à un nombre quelconque (fini ou infini) de feuillets, Comptes Rendue, 148 (1909), 1446.

11. C. Neumann, Berichten der K. Sachs. Gesellschaft der Wissenschaft, April 21 and: October 31, 1870.

12. - Vorlesungen uber Riemanns Theorie, 2nd Edition, Leipzig, 1884.

13. Leo Sario, Alternating method on arbitrary Riemann surfaces, Pacific J. Math., $\mathbf{3}$ : 1953), 631-645.

14. - Minimizing Operators on Subregions, Proc. Amer. Math. Soc., 4 (1953),. $350-355$.

15. - A linear operator method on arbitrary Riemann surfaces, Trans. Amer. Math. Soc. 72, (1952), 281-295.

16. Construction of functions with prescribed properties on Riemann surfaces Ann. Math. Studies, 30 (1953), 63-76.

17. H. A. Schwarz, Gesammelte Mathematische Abhandlungen, Springer, 1890.

18. S. Stoilow, Leçons sur les Principes topologiques de la Théorie des Fonctions Analyti-ques, Gauthier-Villars, 1938.

19. Karl Weierstrass, Mathematische Werke von Karl Weierstrass, Berlin, 1894.

20. Hermann Weyl, Die Idee der Riemannschen Flache, Teubner, 1913. 


\section{PACIFIC JOURNAL OF MATHEMATICS}

\section{EDITORS}

Ralph S. Phillips

Stanford University

Stanford, California

F. H. BrownelL

University of Washington

Seattle 5 , Washington
A. L. Whiteman

University of Southern California Los Angeles 7. California

L. J. PAIGE

University of California

Los Angeles 24, California

ASSOCIATE EDITORS
E. F. BECKENBACH
D. DERRY
H. L. ROYDEN
E. G. STRAUS
T. M. CHERRY
M. OHTSUKA
E. SPANIER
F. WOLF

\section{SUPPORTING INSTITUTIONS}

UNIVERSITY OF BRITISH COLUMBIA

CALIFORNIA INSTITUTE OF TECHNOLOGY

UNIVERSITY OF CALIFORNIA

MONTANA STATE UNIVERSITY

UNIVERSITY OF NEVADA

NEW MEXICO STATE UNIVERSITY

OREGON STATE COLLEGE

UNIVERSITY OF OREGON

OSAKA UNIVERSITY

UNIVERSITY OF SOUTHERN CALIFORNIA
STANFORD UNIVERSITY

UNIVERSITY OF TOKYO

UNIVERSITY OF UTAH

WASHINGTON STATE COLLEGE,

UNIVERSITY OF WASHINGTON

AMERICAN MATHEMATICAL SOCIETY

CALIFORNIA RESEARCH CORPORATION

HUGHES AIRCRAFT COMPANY

SPACE TECHNOLOGY LABORATORIES

NAVAL ORDNANCE TEST STATION

Printed in Japan by International Academic Printing Co., Ltd., Tokyo, Japan

Reprinted 1966 in the United States of America 


\section{Pacific Journal of Mathematics}

\section{Vol. 11, No. $1 \quad$ November, 1961}

A. A. Albert, Generalized twisted fields ............................ 1

Richard Arens, Operational calculus of linear relations ................... 9

John Herbert Barrett, Disconjugacy of a self-adjoint differential equation of the fourth order ....................................... 25

Paul Richard Beesack, Hardy's inequality and its extensions ............... 39

Julius Rubin Blum and David Lee Hanson, On invariant probability measures.

II .............................................

Robert Allen Bonic, Symmetry in group algebras of discrete groups.......... 73

R. Creighton Buck, Multiplication operators ...................... 95

Jack Gary Ceder, Some generalizations of metric spaces ................. 105

Meyer Dwass, Random crossings of cumulative distribution functions ......... 127

Albert Edrei, Wolfgang H. J. Fuchs and Simon Hellerstein, Radial distribution and

deficiencies of the values of a meromorphic function ............... 135

William Cassidy Fox, Harmonic functions with arbitrary local singularities ..... 153

Theodore Thomas Frankel, Manifolds with positive curvature ............... 165

Avner Friedman, A strong maximum principle for weakly subparabolic

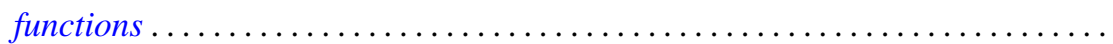

Watson Bryan Fulks and J. O. Sather, Asymptotics. II. Laplace's method for

multiple integrals ......................................

Adriano Mario Garsia and Eugene Richard Rodemich, An embedding of Riemann

surfaces of genus one ..................................... 193

Irving Leonard Glicksberg, Weak compactness and separate continuity......... 205

Branko Grünbaum, On a conjecture of H. Hadwiger .................. 215

Frank J. Hahn, On the action of a locally compact group on $E_{n} \ldots \ldots \ldots \ldots \ldots . . \ldots 221$

Magnus R. Hestenes, Relative hermitian matrices ..................... 225

G. K. Kalisch, On similarity invariants of certain operators in $L_{p} \ldots \ldots \ldots \ldots .247$

Yitzhak Katznelson and Walter Rudin, The Stone-Weierstrass property in Banach

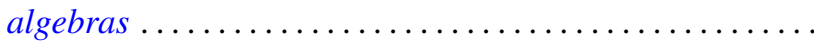

Samir A. Khabbaz, The subgroups of a divisible group $G$ which can be represented as intersections of divisible subgroups of $G \ldots \ldots \ldots \ldots \ldots \ldots \ldots \ldots \ldots . \ldots \ldots 7$

Marvin Isadore Knopp, Construction of a class of modular functions and forms .......................................... 275

Charles Alan McCarthy, Commuting Boolean algebras of projections .......... 295

T. M. MacRobert, Transformations of series of E-functions ................ 309

Heinz Renggli, An inequality for logarithmic capacities ................. 313

M. S. Robertson, Applications of the subordination principle to univalent functions .......................................... 315

David Sachs, Partition and modulated lattices ..................... 325

Frank S. Scalora, Abstract martingale convergence theorems ............... 347

Elbert A. Walker, Torsion endomorphic images of mixed Abelian groups ........ 375

Morgan Ward, The prime divisors of Fibonacci numbers................. 379

Charles R. B. Wright, On the nilpotency class of a group of exponent four....... 387 